\title{
Fast and simple protein-alignment-guided assembly of orthologous gene families from microbiome sequencing reads
}

\author{
Daniel H. Huson ${ }^{1,2^{*}}$, Rewati Tappu', Adam L Bazinet ${ }^{3,4}$, Chao Xie ${ }^{5}$, Michael P. Cummings ${ }^{3}$, Kay Nieselt ${ }^{1}$
} and Rohan Williams ${ }^{2}$

\begin{abstract}
Background: Microbiome sequencing projects typically collect tens of millions of short reads per sample. Depending on the goals of the project, the short reads can either be subjected to direct sequence analysis or be assembled into longer contigs. The assembly of whole genomes from metagenomic sequencing reads is a very difficult problem. However, for some questions, only specific genes of interest need to be assembled. This is then a gene-centric assembly where the goal is to assemble reads into contigs for a family of orthologous genes.

Methods: We present a new method for performing gene-centric assembly, called protein-alignment-guided assembly, and provide an implementation in our metagenome analysis tool MEGAN. Genes are assembled on the fly, based on the alignment of all reads against a protein reference database such as NCBI-nr. Specifically, the user selects a gene family based on a classification such as KEGG and all reads binned to that gene family are assembled.

Results: Using published synthetic community metagenome sequencing reads and a set of 41 gene families, we show that the performance of this approach compares favorably with that of full-featured assemblers and that of a recently published HMM-based gene-centric assembler, both in terms of the number of reference genes detected and of the percentage of reference sequence covered.

Conclusions: Protein-alignment-guided assembly of orthologous gene families complements whole-metagenome assembly in a new and very useful way.
\end{abstract}

Keywords: Sequence assembly, String graph, Functional analysis, Software

\section{Background}

Functional analysis of microbiome sequencing reads-by which we mean either metagenomic or metatranscriptomic shotgun sequencing reads-usually involves aligning the six-frame translations of all reads against a protein reference database such as NCBI-nr [1], using a high-throughput sequence aligner such as DIAMOND [2]. Each read is then assigned to a functional family, such as a KEGG KO group [3] or InterPro family [4], based on the annotation of the most similar protein reference sequence.

\footnotetext{
* Correspondence: Daniel.Huson@uni-tuebingen.de

${ }^{1}$ Center for Bioinformatics, University of Tübingen, Sand 14, 72076 Tübingen, Germany

${ }^{2}$ Life Sciences Institute, National University of Singapore, 28 Medical Drive,

Singapore 117456, Singapore

Full list of author information is available at the end of the article
}

A gene-centric assembly for a family of orthologous genes $F$ is the assembly of all reads associated with $F$. One approach to this is simply to run an existing assembly tool on the reads. In this paper, we present a new approach to gene-centric assembly that we call protein-alignment-guided assembly. We provide an implementation of this approach in the latest release of the metagenomic analysis tool MEGAN Community Edition [5] and will refer to this as the MEGAN assembler. The defining feature of the protein-alignment-guided assembly is that it uses existing protein alignments to detect DNA overlaps between reads. Our implementation of this method is easy to use; it only takes a few mouse clicks to obtain the assembly of any gene family of interest, in contrast to other approaches that require some amount of scripting. 
We compare the performance of the MEGAN assembler to that of several standalone assemblers including IDBAUD [6], Ray [7], and SOAPdenovo [8], and we also compare its performance to that of Xander [9], a gene-centric assembler that employs protein profile HMMs (Hidden Markov Models) rather than sequence alignment to recruit reads. Performance comparisons are based on 41 gene families from a synthetic microbiome community [10].

We use two measures of performance. To assess how well individual gene sequences are assembled, we report the percentage of sequence covered by the longest contig that maps to a given reference sequence. To assess how well gene sequences are detected for different organisms, we report the number of organisms for which the longest mapped contig covers at least half of the corresponding reference sequence.

All assemblers produce similar numbers of contigs and few, if any, false positive contigs. In our evaluation, we find that the MEGAN assembler performs best in terms of the percentage of reference genes covered and percentage of reference gene sequences detected.

\section{Methods}

The main technical contribution of this paper is the design and implementation of a "protein-alignment-guided" assembly algorithm that is explicitly designed for gene-centric assembly. It is integrated in our metagenome analysis program MEGAN and can be launched and run interactively. The algorithm is based on the concept of a string graph [11] and follows the overlap-layout-consensus paradigm [12]. We use existing protein alignments to infer DNA overlaps and provide a simple path-extraction algorithm to layout reads into contigs. As we only consider perfect overlaps, we obtain a consensus sequence for a contig simply by concatenating reads (accounting for overlaps).

Let $F$ be a family of orthologous genes. For example, the KEGG orthology group K03043 represents the DNAdirected RNA polymerase subunit beta, and there are 3216 protein sequences available for this family in the KEGG database. Let $R$ denote the set of all reads that are assigned to $F$ based on a DIAMOND alignment of all reads against a protein reference database such as NCBI-nr or KEGG.
Our assembly approach is based on an overlap graph. Usually, the set of nodes of an overlap graph is given by the set of reads. However, in our construction, we only use an aligned part of each read $r$, which we call the aligned core of $r$. In more detail, we define the aligned core $c(r)$ of any read $r$ to be the segment of the read that is covered by its highest-scoring local alignment to any protein reference sequence in $F$, using the forward or reverse strand of the read, depending on whether the frame of the alignment is positive or negative, respectively.

We build an overlap graph $G=(V, E)$ for $F$ as follows. The set of nodes $V$ consists of the aligned cores of all reads that have at least one significant alignment to a protein reference sequence. Two such nodes $r$ and $s$ are connected by a directed edge $e$ from $r$ to $s$ in $E$, if there exists a protein reference sequence $p \in F$ such that:

1. A suffix of $r$ and a prefix of $s$ each have a significant alignment with $p$;

2. A suffix of the former alignment overlaps a prefix of the latter;

3. The induced gap-free DNA alignment between $r$ and $s$ has perfect identity; and

4. The length of the induced DNA alignment exceeds a specified threshold (20 bp, by default).

We define the weight $\omega(e)$ of any such edge $e$ to be the length of the induced DNA alignment. See Fig. 1 for an illustration of the overlapping process.

One advantage of using the aligned cores of reads, rather than the complete reads, is that this reduces the need to perform quality trimming of reads, as local alignments should not usually extend into stretches of low-quality sequence. The net effect is that reads are filtered and trimmed, not based on some arbitrary quality valuedassociated thresholds as with standard read trimming and filtering procedures, but rather based on the outcome of alignment as protein sequence to reference genes.

We emphasize that overlaps between reads are not only inferred from alignments to just one particular reference sequence (which would be a simple reference-guided assembly), but rather, each read usually participates in

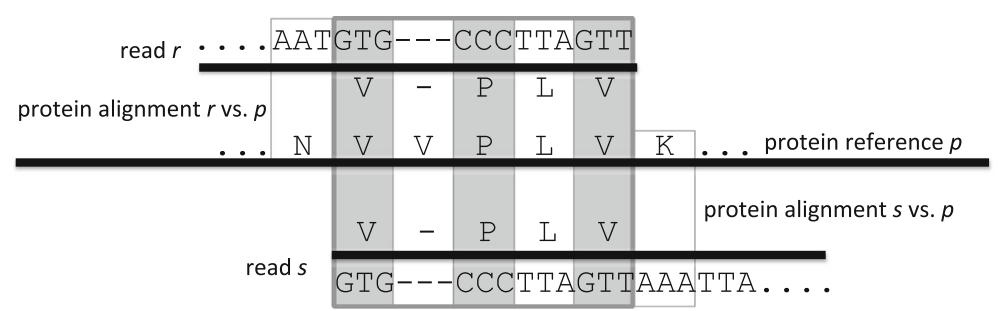

Fig. 1 Induced DNA overlap edges. If two reads $r$ and $s$ both have a protein alignment to the same reference protein $p$, then this defines an overlap edge between the corresponding nodes if the induced DNA alignment has 100\% identity. This induced DNA alignment is of length 12 , as we ignore any induced gaps 
overlaps that are induced by alignments to a number of different reference proteins (typically orthologous proteins from different organisms). There is no need to explicitly reconcile these alignments as they only serve to help detect potential DNA overlaps.

The construction of the overlap graph for a given set of reads and associated alignments to protein references is computationally straightforward to implement. We first build a dictionary mapping each reference sequence to the set of reads that align to it. Then, for each reference sequence, we investigate all pairs of overlapping alignments to determine whether to place an overlap edge between the two nodes representing the corresponding aligned cores. Let $k$ denote the number of reference sequences on which at least two alignments overlap. In the worst case, all $n$ reads align to all $k$ references in an overlapping manner and so the number of edges to add to the overlap graph is at most $\mathrm{O}\left(\mathrm{kn}^{2}\right)$.

Once the overlap graph has been constructed, the task is to extract contigs from the graph, by first identifying paths through the graph and then computing a consensus sequence from the sequences along those paths. When implementing a sequence assembler under the overlap-layout-consensus paradigm, the layout phase, which consists of determining appropriate paths through the overlap graph that will give rise to contigs, is made difficult by repeat-induced cycles and other artifacts in the overlap graph [12].

Cycles appear only very rarely when assembling the reads of a single gene family. Before processing the graph, we break any directed cycle that exists by deleting the lightest edge in the cycle. Thus, the overlap graph is a directed acyclic graph.

Let $P=\left(r_{1}, e_{1}, r_{2}, \ldots, r_{n-1}, \mathrm{e}_{n-1}, r_{n}\right)$ be a directed path of edges in the overlap graph $G$, where $e_{i}$ is the overlap edge between reads $r_{i}$ and $r_{i+1}$ for $i=1, \ldots, n-1$. We define the weight of $P$ as $\omega(P)=\Sigma_{i} \omega\left(e_{i}\right)$ the total number of overlapping nucleotides along the path.

Our layout algorithm operates by repeating the following steps:

1. Determine a path $P$ of maximum weight;

2. Construct a contig $C$ by concatenating all read sequences along $P$, accounting for overlaps;

3. Report $C$ if the contig exceeds a specified threshold for minimum length and/or minimum average coverage;

4. Remove all nodes and edges of $P$ from the graph $G$; and

5. Terminate when no paths remain.

The problem of determining a path of maximum weight in an acyclic-directed graph is solvable in linear time by relaxing vertices in topological order (see [13, p. 661-666]).
We then build a second overlap graph $H$ whose set of nodes consists of all contigs assembled from reads. Any two contigs $c$ and $d$ are connected by a directed edge $(c, d)$ in $H$ if and only if there exists an overlap alignment between a suffix of $c$ and a prefix $d$ of length $\geq 20$ (by default) and percent identity of at least $98 \%$.

First, we use the graph $H$ to identify any contig that is completely contained in a longer one with a percent identity of $98 \%$ or more. Such contained contigs are discarded. This addresses the issue that sequencing errors give rise to shorter contigs that differ from longer ones by a small number of mismatches. We then proceed as described above for $G$ to assemble the remaining contigs into longer ones, if possible. The number of overlap edges is usually very small, and thus, only a small number of contigs are merged.

The latest release of MEGAN provides an implementation of protein-alignment-guided assembly. The program allows the user to import the result of a BLASTX or DIAMOND alignment of a file of reads against a protein reference database and assigns the reads to nodes in a taxonomy and a number of functional classifications (KEGG, SEED [14], eggNOG [15], or InterPro2GO). When importing a BLASTX file or "meganizing" a DIAMOND file, the user must instruct MEGAN to perform the desired functional classifications by selecting the appropriate check boxes and providing appropriate mapping files that map NCBI accession numbers to functional entities, as described in [5]. In addition, we provide a command-line implementation called gc-assembler for use in a non-interactive setting.

The MEGAN assembler can be used in a variety of ways. First, the user can select any node(s) in any of the functional classifications to define the gene family or families to assemble. Clicking the "Export Assembly" menu item will then launch the assembler on each of the selected nodes, and the output will be written to one file per gene family in FASTA format. Second, when viewing the alignments of reads against a particular reference sequence in the MEGAN alignment viewer, the user can launch the assembler for the particular reference sequence. In addition, the user can have the alignment viewer layout reads by their membership in contigs. Figure 2a shows the alignment of the reads against a protein reference sequence in the alignment viewer, and Fig. $2 \mathrm{~b}$ shows the alignment of reads laid out by their membership in contigs.

The main parameters of the assembly algorithm are minOverlapReads, the minimum number of bases that two reads should overlap by; minReads, the minimum number of reads required for a contig; minLength, the minimum length of a contig; minAvCoverage, the minimum average coverage of a contig; minOverlapContigs, the minimum number of bases that two contigs should 
$\mathbf{a}$ Alignment for 'K03043 DNA-directed RNA polymerase subunit beta [EC:2.7.7.6]' - Synthetic-Shakky-2013.daa - MEGAN

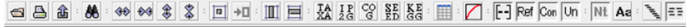

List of 1124 avallable reference sequences for 'K03043 DNA-directed RNA polymerase subunit beta [EC:2.7.7.6]' (double click on one to see alignment):

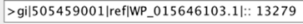
Alignment for reference sequence 'sgi|4954674121|ref|WP_008192102.1|'

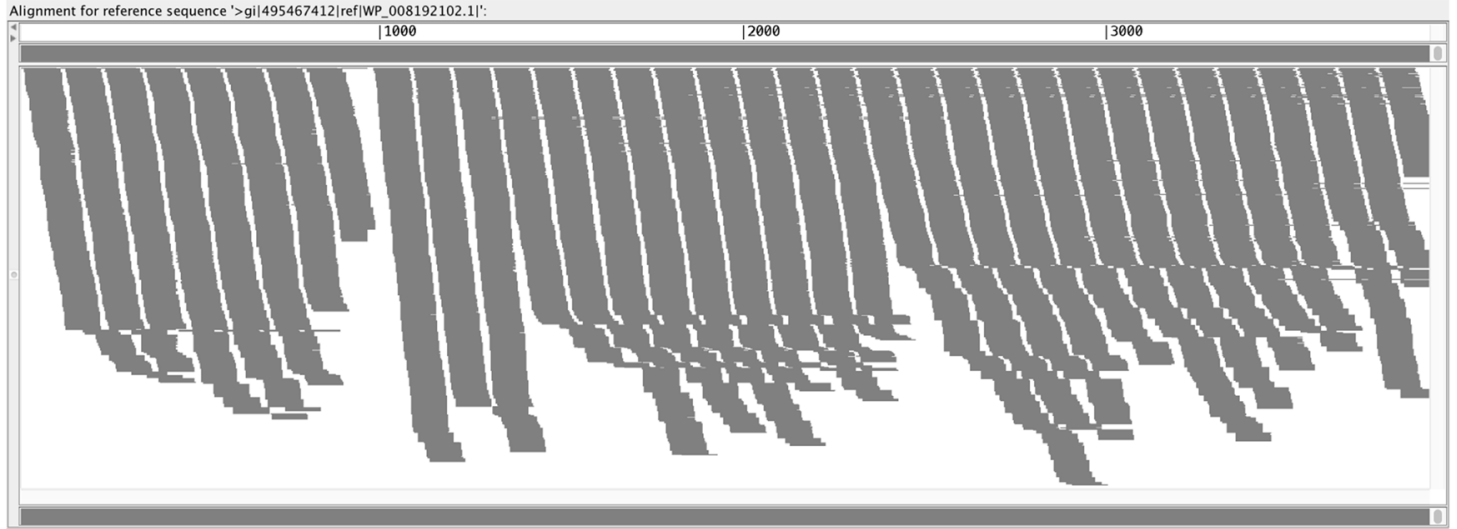

Alignnent: $13279 \times 3987$

3.0 of 10.46

b Alignment for 'K03043 DNA-directed RNA polymerase subunit beta [EC:2.7.7.6]' - Synthetic-Shakya-2013.daa - MEGAN

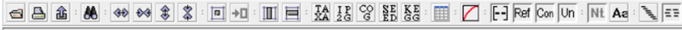

List of 1124 available reference sequences for 'K03043 DNA-directed RNA polymerase subunit beta IEC 2.7.7 61' (double click on one to see alignment):$>$ gil $505459001 \mid$ refilip_015646103.1|: 13279

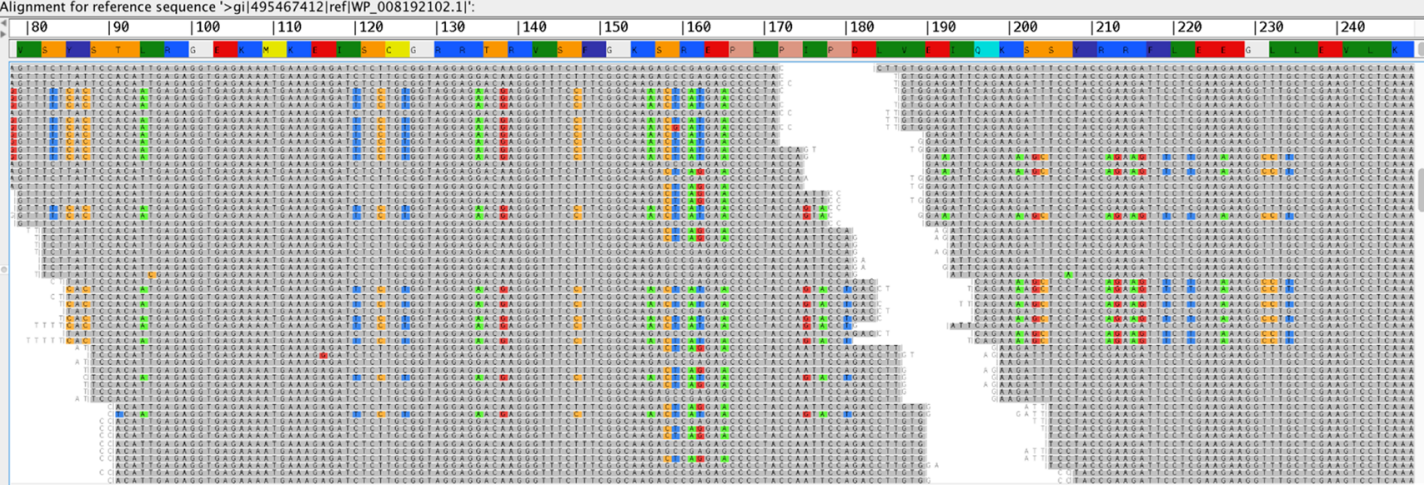

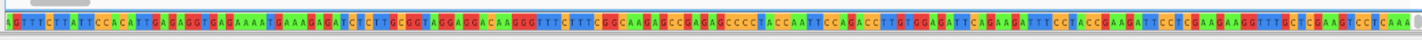
Algmment $13279 \times 3897$

C Alignment for 'K03043 DNA-directed RNA polymerase subunit beta [EC:2.7.7.6]' - Synthetic-Shakya-2013.daa - MEGAN

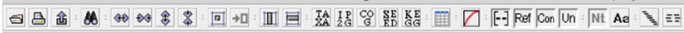

List of 1124 aval able reference sequences for 'K03043 DNA-directed RNA polymerase subunit beta [EC: 2.7.7.6.' (double click on one to see aligenent)

$>$ gi|1505459001|reflWP_.015646103.11:: 13279

Âlignment for reference sequence ' $>$ gil4954667412| |refiWP 008192102.11'.

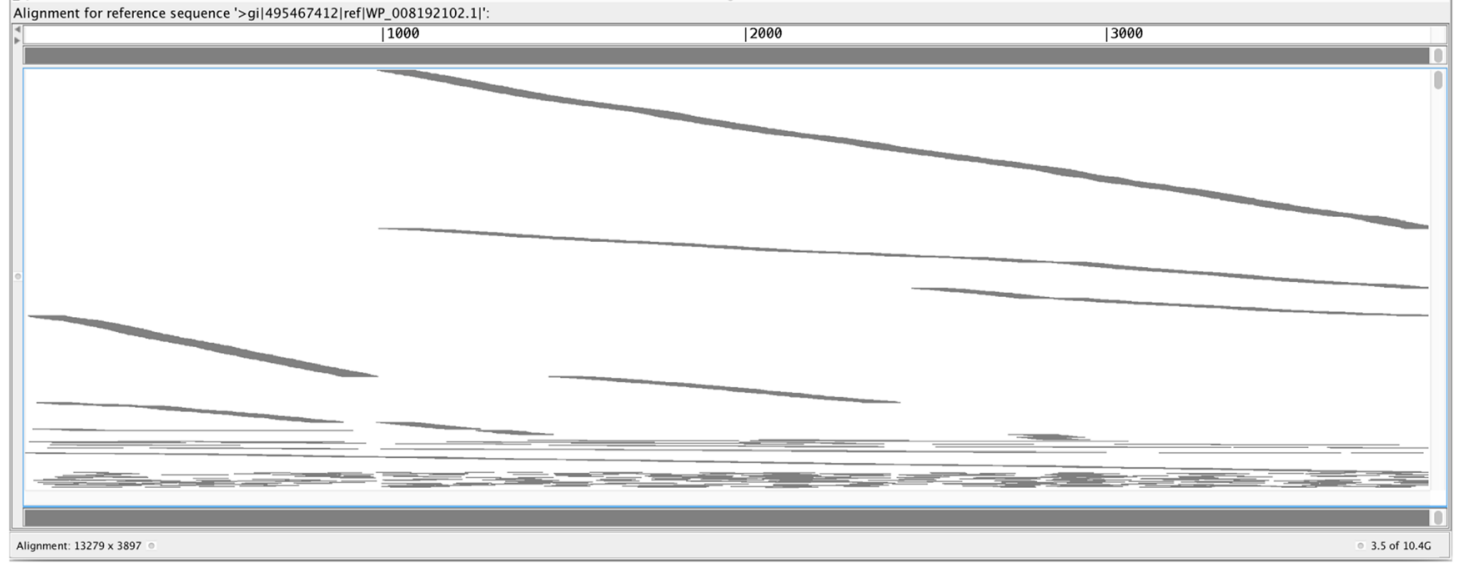

Fig. 2 Alignment viewer. a Alignment of 13,623 reads against one of the reference sequences representing bacteria rpoB, as displayed in MEGAN's alignment viewer. $\mathbf{b}$ More detailed view in which nucleotides that do not match the consensus sequence are highlighted in color. c Reads are ordered by contig membership and decreasing length of contigs 
overlap by to be merged; and minPercentIdentityContigs, the minimum percent identity at which contigs are deemed to overlap or be contained.

To assemble a given gene, MEGAN first needs to extract the associated reads and alignments from the indexed file containing the full set of reads and alignments. This usually takes a couple of minutes. Once this step has been completed, protein-alignment-guided assembly of the reads will take on the order of seconds to minutes (see below).

\section{Experimental evaluation}

We downloaded a dataset of 108 million Illumina reads (54 million per paired-end file) obtained from sequencing a synthetic community containing 48 bacterial species and 16 archaeal species (SRA run SRR606249; [10]). The read length is $101 \mathrm{bp}$. We aligned these reads against the NCBI-nr database (downloaded February 2015) using DIAMOND (E value cutoff of 0.01), which resulted in more than one billion alignments, involving 87 million reads. We ran the resulting DIAMOND file through our program daa-meganizer, which performs taxonomic and functional assignment of all reads in the dataset based on the alignments found by DIAMOND, and then appends the resulting classifications and indices to the DIAMOND file. This "meganized" DIAMOND file is publicly accessible in MEGAN via MeganServer.

We based our experimental analysis on a set of singlecopy phylogenetic marker genes [16] so as to simplify the task of evaluating the performance of the different methods. We also consider some other genes, archaeal and bacterial rpoB, cheA, ftsZ, and atoB, to see how the assembly methods perform on other types of genes.

For each gene family in the study, we determined the corresponding KEGG orthology (KO) group and ran the MEGAN assembler on all reads assigned to that family. The assembler was run with the default options of minOverlapReads $=20, \quad$ minReads $=2, \quad$ minLength $=200$, minOverlapContigs $=20$, and minPercentIdentityContigs $=98$. In addition, for each $\mathrm{KO}$ group, we saved all assigned reads to a file and then assembled them using IDBA-UD, Ray, and SOAPdenovo. All assemblers were run with default options.

To evaluate Xander, we downloaded all associated amino acid and nucleotide KEGG gene sequences for the 41 gene families, aligned the amino acid sequences with MAFFT (using the -auto option; version 7.187 [17, 18]), and built HMMs and configured supporting files according to Xander documentation.

Running Xander using default parameters (min_bits = 50 and min_length $=150$ ) gave rise to small number of contigs per gene family that was much lower than the number of gene family members in the community, resulting in an unacceptable number of false negatives. To address this, we experimented with different parameter settings until Xander produced a number of contigs that is similar to that produced by the other four assemblers. We used the following parameters for Xander: min_bits $=1$, min_length $=1$, filter_size $=39$, min_count $=1$, and max_jvm_heap $=64$ GB.

Table 1 shows the number of reads assigned to each gene family, as well as the number of reference gene DNA sequences that represent each gene family.

For a typical gene family with $\approx 20,000$ assigned reads, the MEGAN assembler took approximately $30 \mathrm{~s}$ to run, while for other assemblers the time taken was as follows: $55 \mathrm{~s}$ (IDBA-UD), $75 \mathrm{~s}$ (Ray), and $3 \mathrm{~s}$ (SOAPdenovo), on a server with 32 cores. Xander took approximately $1 \mathrm{~h}$ (excluding the time required to build the de Bruijn graph) to run on a server with 20 cores. Maximum memory usage was set to 12 GB for the MEGAN assembler and $64 \mathrm{~GB}$ for Xander, whereas the other assemblers used 1.5-3 GB of memory.

Using a minimum contig length of $200 \mathrm{bp}$, all assemblers produced a similar average number of contigs per gene family: 73 (MEGAN), 48 (IDBA-UD), 69 (Ray), 78 (SOAPdenovo), and 93 (Xander). The number of contigs produced per gene family is shown in Fig. 3.

To allow an analysis of the performance of the different methods, we aligned all contigs to all reference genes associated with the synthetic community using BLASTN. In nearly all cases, we found an alignment of at least $98 \%$ identity to a reference organism that was part of the synthetic community. In the few remaining cases, a high-identity BLASTX alignment of at least $98 \%$ identity to the corresponding protein sequence was found. In nearly all cases, the alignments covered at least $99 \%$ of the contig. This indicates that there are only very few, if any, false positive contigs.

To assess assembly performance, for each assembly, each gene family and each species in the synthetic community that contains a member of the gene family, we determined the percentage of reference gene sequence covered by the longest contig aligned to it (Fig. 4). In this calculation, only matching bases are counted.

To assess detection performance, we consider a reference gene sequence to be successfully detected by an assembler if the longest contig covers $50 \%$ or more of the sequence, again, counting only matching bases. In Table 1 , for each gene and each assembler, we report the number of reference sequences detected (as defined above) and provide a summary of the proportion of reference sequences detected per gene family in Fig. 5, showing that the protein-alignmentbased assembler implemented in MEGAN performs best for most genes. 
Table 1 For each gene family studied, we report the KEGG orthology group, number of reads assigned to that group by DIAMOND, number of reference gene sequences that exist in the synthetic community, and number of reference genes "detected" by each method: MEGAN, IDBA-UD, Ray, SOAPdenovo, and Xander

\begin{tabular}{|c|c|c|c|c|c|c|c|c|}
\hline Gene family & KEGG & Reads & References & MEGAN & IDBA-UD & Ray & SOAP & Xander \\
\hline Acetyl-CoA C-acetyltransferase & K00626 & 58,135 & 64 & 31 & 16 & 12 & 12 & 23 \\
\hline Archael rpoB1 & K03044 & 17,875 & 16 & 7 & 7 & 6 & 3 & 9 \\
\hline Archael rpoB2 & K03045 & 12,025 & 16 & 8 & 8 & 6 & 5 & 5 \\
\hline Cell division protein & K03531 & 45,881 & 48 & 37 & 39 & 12 & 7 & 12 \\
\hline Bacterial rpoB & K03043 & 105,212 & 64 & 43 & 16 & 12 & 13 & 50 \\
\hline Phenylalanyl-tRNA synthetase alpha subunit & K01889 & 44,779 & 64 & 57 & 56 & 47 & 51 & 48 \\
\hline Phenylalanyl-tRNA synthetase beta subunit & K01890 & 73,072 & 64 & 53 & 50 & 42 & 38 & 35 \\
\hline Phosphoribosylformylglycinamidine cyclo ligase & K01933 & 31,919 & 64 & 58 & 59 & 46 & 45 & 54 \\
\hline Ribonuclease HII & K03470 & 18,707 & 64 & 54 & 55 & 53 & 45 & 48 \\
\hline Ribosomal protein L1 & K02863 & 24,190 & 64 & 57 & 49 & 45 & 48 & 53 \\
\hline Ribosomal protein L10 & K02864 & 23,970 & 64 & 58 & 48 & 55 & 57 & 55 \\
\hline Ribosomal protein L11 & K02867 & 17,113 & 64 & 60 & 50 & 51 & 60 & 59 \\
\hline Ribosomal protein L13 & K02871 & 17,642 & 64 & 58 & 54 & 53 & 57 & 45 \\
\hline Ribosomal protein L14 & K02874 & 13,435 & 64 & 56 & 42 & 49 & 58 & 60 \\
\hline Ribosomal protein L15 & K02876 & 13,087 & 64 & 59 & 56 & 50 & 55 & 55 \\
\hline Ribosomal protein L16 & K02878 & 10,058 & 64 & 46 & 34 & 36 & 44 & 44 \\
\hline Ribosomal protein L18 & K02881 & 14,856 & 64 & 57 & 48 & 56 & 57 & 55 \\
\hline Ribosomal protein L2 & K02886 & 29,849 & 64 & 60 & 54 & 46 & 55 & 57 \\
\hline Ribosomal protein L22 & K02890 & 15,875 & 64 & 59 & 54 & 55 & 57 & 51 \\
\hline Ribosomal protein L24 & K02895 & 11,786 & 64 & 60 & 46 & 56 & 58 & 44 \\
\hline Ribosomal protein L25 & K02897 & 12,941 & 64 & 41 & 41 & 39 & 42 & 41 \\
\hline Ribosomal protein L29 & K02904 & 4913 & 64 & 29 & 8 & 33 & 34 & 8 \\
\hline Ribosomal protein L3 & K02906 & 30,192 & 64 & 59 & 47 & 51 & 57 & 51 \\
\hline Ribosomal protein L4 & K02926 & 14,539 & 64 & 44 & 41 & 39 & 43 & 44 \\
\hline Ribosomal protein L5 & K02931 & 20,533 & 64 & 60 & 58 & 55 & 59 & 58 \\
\hline Ribosomal protein L6 & K02933 & 20,645 & 64 & 58 & 41 & 56 & 59 & 60 \\
\hline Ribosomal protein S10 & K02946 & 11,327 & 64 & 56 & 42 & 48 & 56 & 54 \\
\hline Ribosomal protein S11 & K02948 & 10,793 & 64 & 47 & 43 & 51 & 52 & 56 \\
\hline Ribosomal protein S12 & K02950 & 14,199 & 64 & 61 & 41 & 48 & 60 & 58 \\
\hline Ribosomal protein S13 & K02952 & 13,975 & 64 & 59 & 46 & 56 & 60 & 58 \\
\hline Ribosomal protein S15 & K02956 & 10,795 & 64 & 54 & 16 & 43 & 55 & 50 \\
\hline Ribosomal protein S17 & K02961 & 10,235 & 64 & 58 & 36 & 49 & 44 & 60 \\
\hline Ribosomal protein S19 & K02965 & 12,479 & 64 & 59 & 39 & 51 & 59 & 58 \\
\hline Ribosomal protein S2 & K02967 & 25,926 & 64 & 61 & 46 & 41 & 53 & 48 \\
\hline Ribosomal protein S3 & K02982 & 25,722 & 64 & 59 & 46 & 48 & 57 & 57 \\
\hline Ribosomal protein S5 & K02988 & 21,761 & 64 & 59 & 55 & 53 & 53 & 56 \\
\hline Ribosomal protein S7 & K02992 & 20,520 & 64 & 60 & 42 & 54 & 60 & 61 \\
\hline Ribosomal protein S8 & K02994 & 14,543 & 64 & 62 & 57 & 58 & 60 & 57 \\
\hline Ribosomal protein S9 & K02996 & 12,927 & 64 & 59 & 52 & 52 & 58 & 61 \\
\hline
\end{tabular}


Table 1 For each gene family studied, we report the KEGG orthology group, number of reads assigned to that group by DIAMOND, number of reference gene sequences that exist in the synthetic community, and number of reference genes "detected" by each method: MEGAN, IDBA-UD, Ray, SOAPdenovo, and Xander (Continued)

\begin{tabular}{|c|c|c|c|c|c|c|c|c|}
\hline Signal recognition particle protein & K03110 & 27,386 & 64 & 35 & 48 & 36 & 19 & 46 \\
\hline Two-component system & K03407 & 47,904 & 64 & 29 & 17 & 15 & 15 & 27 \\
\hline Mean absolute deviation & & & & 9.34 & 19.73 & 18.24 & 15.41 & 14.17 \\
\hline
\end{tabular}

Best results are shown in bold. Mean absolute deviation between the number of references genes and the number detected by each method is reported as a summary statistic

\section{Results and Conclusions}

The work reported on in this paper provides simple and fast access to assemblies of individual gene families from within MEGAN, a popular microbiome sequence analysis tool. Protein-alignment-guided assembly makes use of pre-computed protein alignments to perform genecentric assembly. Alternative ways of performing genecentric assembly include running an external assembly tool on the reads assigned to a specific gene family or using an HMM-based framework such as Xander for read recruitment and assembly.

Based on percent coverage by longest contig and number of gene sequences detected, the MEGAN assembler performs best in our experimental study (Figs. 4 and 5). The average percent coverage values over all gene families are 75.4\% (MEGAN), 62.4\% (IDBA-UD), 64.6\% (Ray), 67.8\% (SOAPdenovo), and 69.6\% (Xander).
Figure 4 indicates that all approaches have difficulties assembling ribosomal protein L29. The reason for this is that members of this gene family are very short, less than 70 aa in many cases, and so the resulting contigs rarely exceed the 200-bp length threshold that we use.

All assembly methods fail on the species Sulfurihydrogenibium yellowstonense. In our analysis, only approximately 46,000 (of 108 million) reads are classified as coming from this species and so this species is represented by substantially less reads than the other species in the mock community. The NCBI-nr database contains 1700 reference proteins for this species, and so, the average number of reads assigned to each protein is only 27 . In addition, none of these reference sequences is annotated by one of the 41 KOs (KEGG orthology groups) used in this study. So, the failure is due to a combination of low coverage

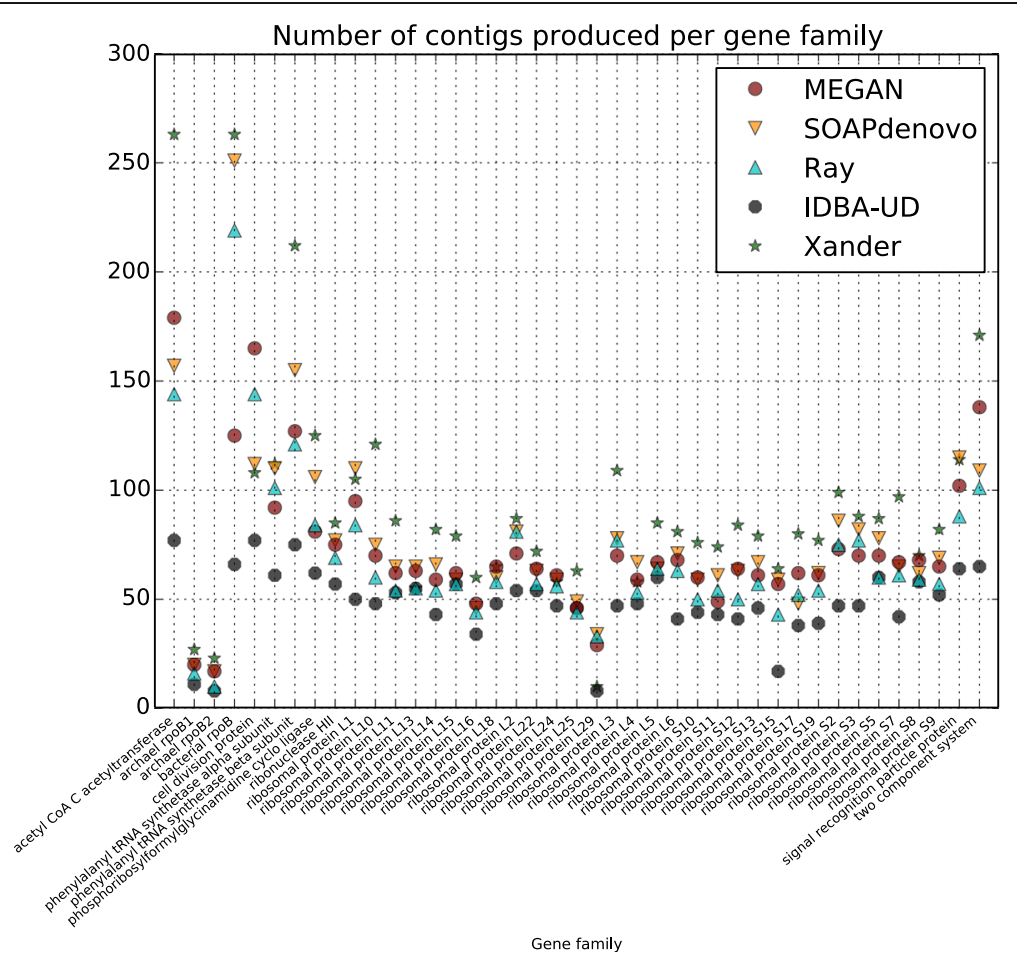

Fig. 3 Summary of number of contigs produced. For each gene family along the $x$-axis, we plot the number of contigs of length $\geq 200$ bp produced by each assembler 


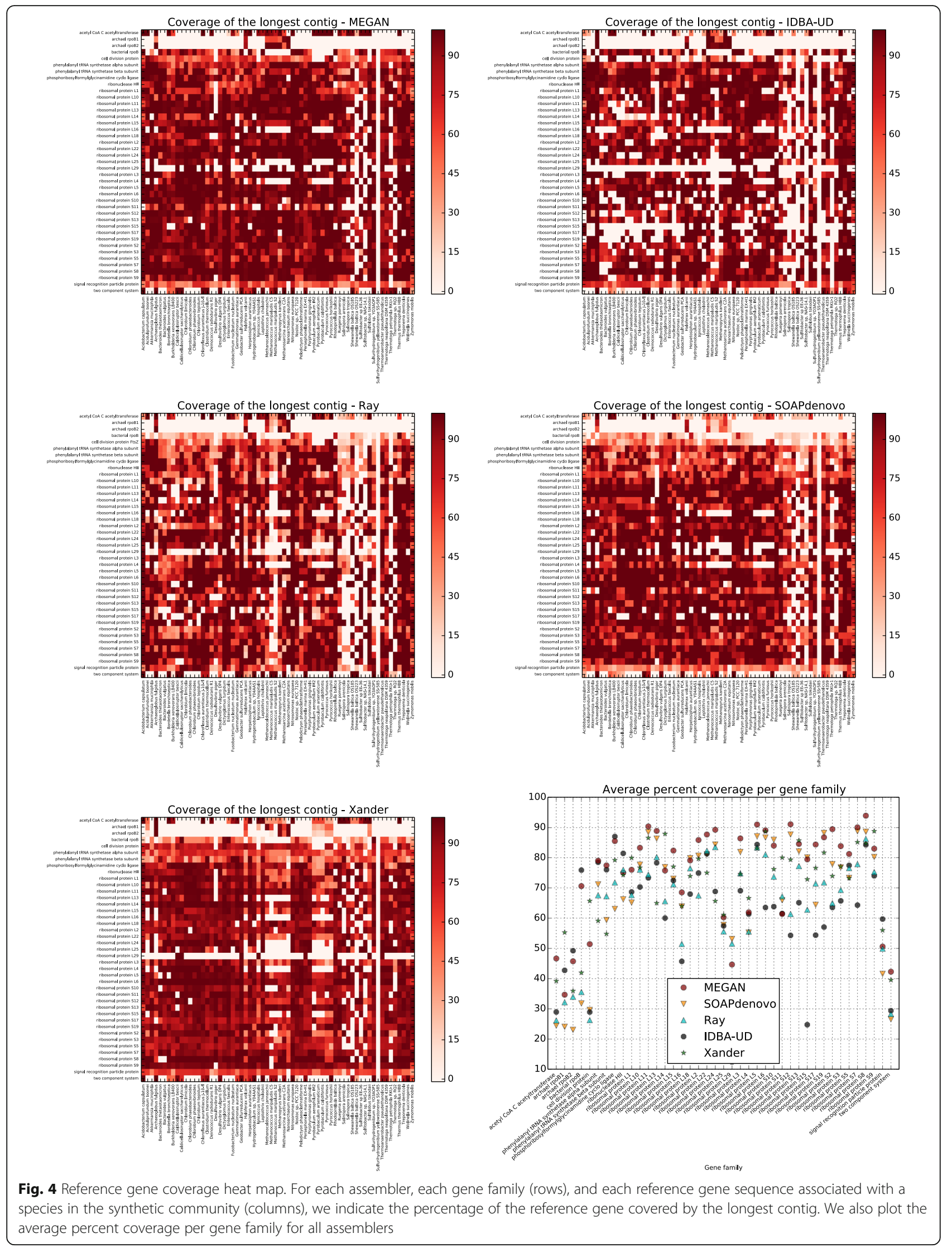




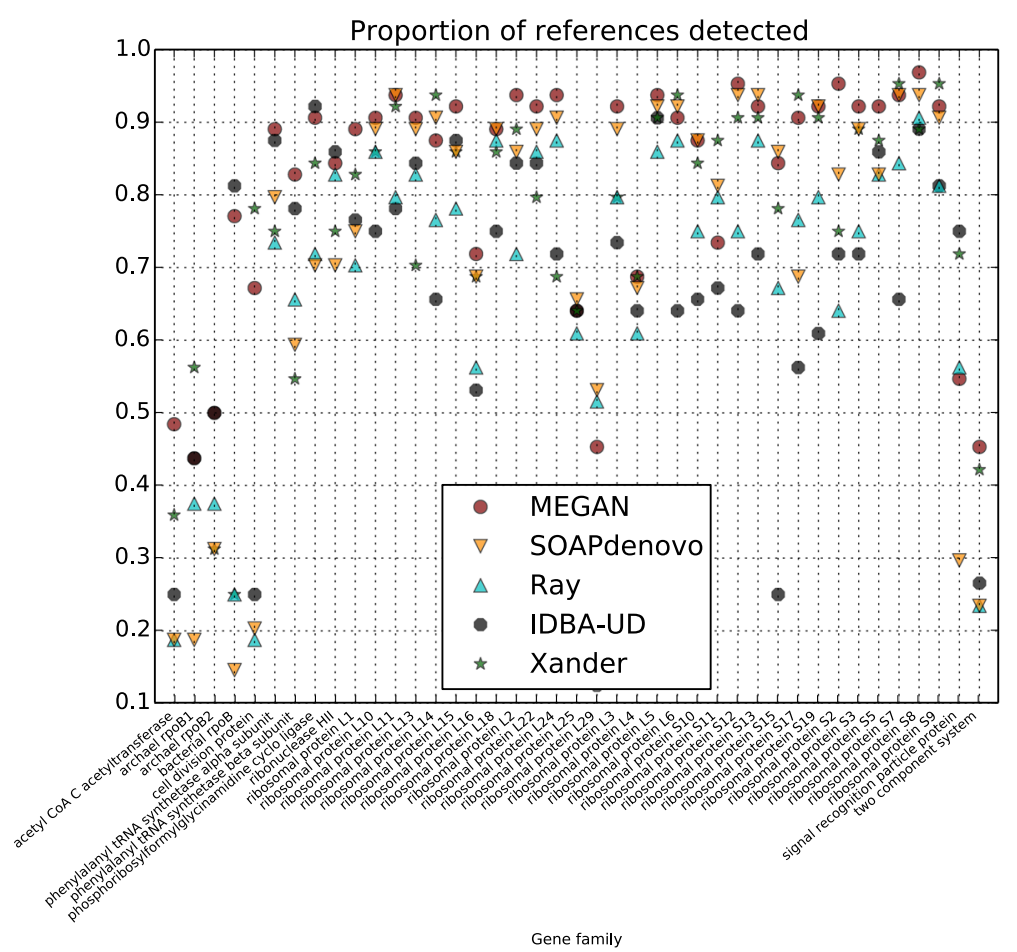

Fig. 5 Reference gene coverage summary. For each gene family along the $x$-axis, we plot the number of reference gene sequences detected by each method

and the absence of any directly corresponding reference sequences.

Note, however, that there are a number of other species (Bacteroides vulgatus, Desulfovibrio piger, Gemmatimonas aurantiaca, and Salinispora arenicola) for which there is no directly corresponding reference sequence for any of the $41 \mathrm{KOs}$, yet for these, the gene-centric assembly appears to work well. In particular, we emphasize that for Gemmatimonas aurantiaca, the closest species that has reference proteins for (any and all of) the $41 \mathrm{KOs}$ is Gemmatirosa kalamazoonesis, which belongs to a different genus.

In our performance analysis, we assign each contig to at most one organism in the synthetic community. However, in some cases, the same contig aligns equally well to the gene reference sequence of two closely related organisms and as a consequence, the analysis reported in Fig. 4 slightly under-predicts the true performance of all methods. This happens, for example, for ribosomal protein L16 for Thermotoga sp. RQ2 and Thermotoga petrophila $R K U-1$. We use a threshold of $98 \%$ identity to determine whether contigs generated by our assembler are deemed to be containing or overlapping each other. This is to ensure that the number of contigs produced by our assembler is similar to that produced by the other approaches. Increasing the threshold to $99 \%$ produces a handful of additional correct gene detections, while roughly doubling the number of contigs.

Gene-centric assembly does not replace the computation of a full assembly of all reads, which remains a challenging problem [19], with some recent advances [20]. DIAMOND alignment of all 108 million reads in the synthetic community [10] against the NCBI-nr database, followed by the gene-centric assembly of all 2834 detected KEGG families using MEGAN, took only one and a half days on a single 32-core server. In contrast, assembly of all 108 million reads from the described synthetic community [10] using Ray-2.3.1 took 6 days on the same server. The resulting assembly contains 52,821 contigs of length $\geq 200 \mathrm{bp}$, with a median length of $802 \mathrm{bp}$, mean length of $3789 \mathrm{bp}$, and maximum length of $600,408 \mathrm{bp}$. We estimate that running Xander on all 2834 KEGG families present in the synthetic community will take 10-100 days on a single server with 20 cores.

We emphasize that protein-alignment-guided assembly as currently implemented in MEGAN only constructs contigs that span known protein domains and so interspersed unknown domains will result in contig fragmentation. Here, the application of a full-featured assembler to all protein-alignment-recruited reads and their mates may result in longer contigs that cover some of the unknown domains. 
As an all-in-one GUI-based desktop application, MEGAN is especially designed for use by biologists and medical researchers that have limited bioinformatics skills. The built-in assembler now provides such users with simple access to sequence assembly techniques on a gene-by-gene basis.

\section{Acknowledgements}

We thank Alexander Seitz for helpful discussions.

\section{Funding}

This work was supported by the Graduate School of the University of Maryland, College Park, and the Institutional Strategy of the University of Tübingen (Deutsche Forschungsgemeinschaft, ZUK 63) and by the Life Sciences Institute of the National University of Singapore.

\section{Availability of data and materials}

Our implementation of protein-alignment-guided assembly presented in this paper is available in the Community Edition of MEGAN, which may be downloaded here: http://ab.inf.uni-tuebingen.de/software/megan6.

The alignments computed by DIAMOND on the synthetic community [10] can be accessed from within MEGAN by connecting to the public

MeganServer database and opening the file named Other/Synthetic-Shakya2013.daa.

\section{Authors' contributions}

All authors contributed to the study design. DHH implemented the proteinalignment-guided assembly algorithm in MEGAN. RT and ALB ran all assemblers and analyzed their performance. DHH and MPC wrote the manuscript. All authors read and approved the final manuscript.

\section{Competing interests}

The authors declare that they have no competing interests.

\section{Consent for publication}

Not applicable.

\section{Ethics approval and consent to participate}

Not applicable.

\section{Author details}

${ }^{1}$ Center for Bioinformatics, University of Tübingen, Sand 14, 72076 Tübingen, Germany. ${ }^{2}$ Life Sciences Institute, National University of Singapore, 28 Medical Drive, Singapore 117456, Singapore. ${ }^{3}$ Center for Bioinformatics and Computational Biology, University of Maryland, 8314 Paint Branch Drive, College Park, MD 20742, USA. ${ }^{4}$ National Biodefense Analysis and Countermeasures Center, 8300 Research Plaza, Fort Detrick, Frederick, MD 21702, USA. ${ }^{5}$ Human Longevity Inc., Singapore, Singapore.

Received: 12 September 2016 Accepted: 17 January 2017 Published online: 25 January 2017

\section{References}

1. Wheeler DL, Tanya B, Benson DA, Bryant SH, Kathi C, Vyacheslav C, Church DM, Michael D, Ron E, Scott F, Michael F, Geer LY, Wolfgang H, Yuri K, Oleg K, David L, Lipman DJ, Madden TL, Maglott DR, Vadim M, James O, Pruitt KD, Schuler GD, Shumway M, Sequeira E, Sherry ST, Sirotkin K, Souvorov A, Starchenko G, Tatusov RL, Tatusova TA, Wagner L, Yaschenko E. Database resources of the National Center for Biotechnology Information. Nucleic Acids Res. 2008;36:D13-21.

2. Buchfink $B$, Xie $C$, Huson DH. Fast and sensitive protein alignment using DIAMOND. Nat Methods. 2015;12:59-60

3. Kanehisa M, Goto S. KEGG: Kyoto Encyclopedia of Genes and Genomes. Nucleic Acids Res. 2000;28(1):27-30.

4. Mitchell A, Chang H-Y, Daugherty L, Fraser M, Hunter S, Lopez R, McAnulla C, McMenamin C, Nuka G, Pesseat S, Sangrador-Vegas A, Scheremetjew M, Rato C, Yong SY, Bateman A, Punta M, Attwood TK, Sigrist CJA, Redaschi N, Rivoire C, Xenarios L, Kahn D, Guyot D, Bork P, Letunic I, Gough J, Oates M, Haft D, Huang H, Natale DA, Wu CH, Orengo C, Sillitoe I, Huaiyu M, Thomas
PD, Finn RD. The InterPro protein families database: the classification resource after 15 years. Nucleic Acids Res. 2015;43(D1):D213-21.

5. Huson DH, Beier S, Flade I, Górska A, El-Hadidi M, Mitra S, Ruscheweyh HJ, Rewati T. MEGAN Community Edition-interactive exploration and analysis of large-scale microbiome sequencing data. PLoS Comput Biol. 2016;12(6): e1004957. doi:10.1371/journal.pcbi.1004957.

6. Yu P, Leung HCM, Yiu SM, Chin FYL. IDBA-UD: a de novo assembler for single-cell and metagenomic sequencing data with highly uneven depth. Bioinformatics. 2012;28(11):1420-8.

7. Boisvert $\mathrm{S}$, Laviolette $F$, Corbeil J. Ray: simultaneous assembly of reads from a mix of high-throughput sequencing technologies. J Comput Biol. 2010;17(11):1519-33

8. Luo R, Liu B, Xie Y, Li Z, Huang W, Yuan J, He G, Chen Y, Pan Q, Liu Y, Tang J, Wu G, Zhang H, Shi Y, Liu Y, Yu C, Wang B, Lu Y, Han C, Cheung DW, Yiu SM, Peng S, Xiaogian Z, Liu G, Liao X, Li Y, Yang H, Wang J, Lam TW, Wang J. SOAPdenovo2: an empirically improved memory-efficient short-read de novo assembler. GigaScience. 2012;1 (1):18.

9. Wang Q, Fish JA, Gilman M, Sun Y, Brown CT, Tiedje JM, Cole JR. Xander: employing a novel method for efficient gene-targeted metagenomic assembly. Microbiome. 2015;3(1):1-13.

10. Shakya M, Quince C, Campbell JH, Yang ZK, Schadt CW, Podar M. Comparative metagenomic and rRNA microbial diversity characterization using archaeal and bacterial synthetic communities. Environ Microbiol. 2013;15(6):1882-99.

11. Myers EW. The fragment assembly string graph. Bioinformatics. 2005;21 suppl 2:ii79-85.

12. Myers EW, Sutton GG, Delcher AL, Dew IM, Fasulo DP, Flanigan MJ, Kravitz SA, Mobarry CM, Reinert KHJ, Remington KA, Anson EL, Bolanos RA, Chou H-H, Jordan CM, Halpern AL, Lonardi S, Beasley EM, Brandon RC, Chen L, Dunn PJ, Lai Z, Liang Y, Nusskern DR, Zhan M, Zhang Q, Zheng X, Rubin GM, Adams MD, Venter JC. A whole-genome assembly of Drosophila. Science. 2000;287:2196-204.

13. Kevin Daniel Sedgewick, Robert; Wayne. Algorithms. Addison-Wesley Professional, fourth edition, 2011.

14. R Overbeek, R Olson, GD Pusch, GJ Olsen, JJ Davis, T Disz, RA Edwards, S Gerdes, B Parrello, M Shukla, V Vonstein, AR Wattam, F Xia, and R Stevens. The SEED and the Rapid Annotation of microbial genomes using Subsystems Technology (RAST). Nucleic Acids Res. 2013;42(Database issue): D206-D214.

15. Powell S, Szklarczyk D, Trachana K, Roth A, Kuhn M, Muller J, Arnold R, Rattei T, Letunic I, Doerks T, Jensen LJ, von Mering C, Bork P. eggNOG v3.0: orthologous groups covering 1133 organisms at 41 different taxonomic ranges. Nucleic Acids Res. 2012;40(Database-Issue):284-9.

16. Wu D, Jospin G, Eisen JA. Systematic identification of gene families for use as "Markers" for phylogenetic and phylogeny-driven ecological studies of bacteria and archaea and their major subgroups. PLoS One. 2013;8:10.

17. Katoh K, Misawa K, Kuma K, Miyata T. MAFFT: a novel method for rapid multiple sequence alignment based on fast Fourier transform. Nucleic Acids Res. 2002;30(14):3059-66.

18. Katoh K, Standley DM. MAFFT multiple sequence alignment software version 7: improvements in performance and usability. Mol Biol Evol. 2013;30(4):772-80

19. Treangen T, Koren S, Sommer D, Liu B, Astrovskaya I, Ondov B, Darling A, Phillippy A, Pop M. MetAMOS: a modular and open source metagenomic assembly and analysis pipeline. Genome Biol. 2013;14(1):R2.

20. Howe AC, Jansson JK, Malfatti SA, Tringe SG, Tiedje JM, Brown CT. Tackling soil diversity with the assembly of large, complex metagenomes. Proc Nat Acad Sci USA. 2014;111(13):4904-9. 